Western University

Scholarship@Western

Chemistry Publications

Chemistry Department

$6-22-2015$

Synthesis of Tetrahydro-1,2-oxazines and

Pyrrolidines via Cycloadditions of Donor-Acceptor Cyclobutanes and Nitrosoarenes

Naresh Vemula

Brian L Pagenkopf

Follow this and additional works at: https://ir.lib.uwo.ca/chempub

Part of the Chemistry Commons

Citation of this paper:

Vemula, Naresh and Pagenkopf, Brian L, "Synthesis of Tetrahydro-1,2-oxazines and Pyrrolidines via Cycloadditions of DonorAcceptor Cyclobutanes and Nitrosoarenes" (2015). Chemistry Publications. 41.

https://ir.lib.uwo.ca/chempub/41 


\title{
Synthesis of Tetrahydro-1,2-oxazines and Pyrrolidines via Cycloadditions of Donor-Acceptor Cyclobutanes and Nitrosoarenes
}

\author{
Naresh Vemula ${ }^{[a]}$ and Brian L. Pagenkopf ${ }^{\star[a]}$
}

Keywords: Donor-Acceptor Cyclobutanes / Cycloadditions / Nitrosoarenes / Lewis Acid Catalysis / Tetrahydro-1,2-oxazines / Pyrrolidines

During efforts to expand the scope of Lewis acid catalyzed [4+2] cycloaddition between donor-acceptor cyclobutanes and nitrosoarenes, an unexpected formation of pyrrolidine products was discovered when $50 \mathrm{~mol} \%$ of $\mathrm{Mgl}_{2}$ was used as a Lewis acid. It was also observed that the electronics of the nitrosoarene and judicious selection of the Lewis acid catalyst have a profound effect on the regioselectivity of the reaction.

\section{Introduction}

Cycloaddition chemistry is an excellent method to construct highly complex structures from simple starting materials. [1] In particular, 1,3-dipolar cycloadditions using a variety of dipolarophiles have been extensively studied and well documented. ${ }^{[2]}$ Interestingly, generation of 1,4-dipolar intermediates by exploitation of ring strain in carbocycles is a clever approach in modern organic synthesis. ${ }^{[3]}$ In this regard, reactivity of donor-acceptor (DA) cyclobutanes has recently garnered significant attention, and a number of reactive partners have been found to undergo cycloadditions with DA cyclobutanes ${ }^{[4]}$ and cyclobutanones. ${ }^{[5]}$

Recently, we reported the first examples of [4+2] cycloadditions between DA cyclobutanes and nitrosoarenes (Scheme 1). ${ }^{[6]}$ The reactions proceed well with electron-neutral and electron-deficient nitrosoarenes to form tetrahydro-1,2-oxazines with excellent regioselectivity; however, no reaction was observed with electron-rich nitrosoarenes under the reported conditions.

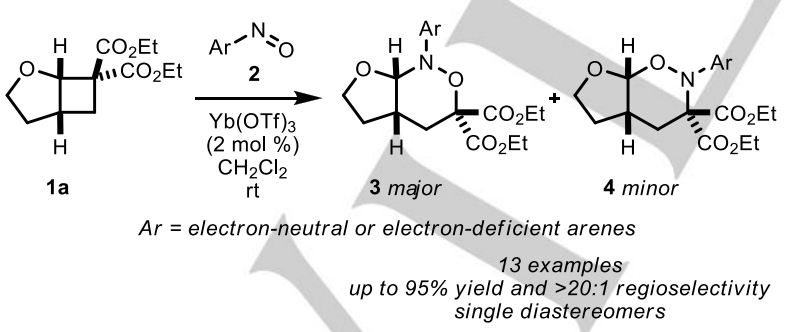

Scheme 1. Previous work with DA cyclobutanes and nitrosoarenes.

[a] Department of Chemistry

The University of Western Ontario

1151 Richmond Street, London, Ontario N6A 5B7, Canada

Tel: +1(519) 6612111 Extn. 81430

Fax: +1(519) 661-3022

E-mail: bpagenko@uwo.ca

Supporting information for this article is available on the WWW under http://www.eurjoc.org/ or from the author

\section{Results \& Discussion}

In an attempt to extend the reaction scope to include electronrich substrates, we employed 1-methoxy-4-nitrosobenzene $\mathbf{2 a}$ as a model substrate. After a thorough screening of Lewis acids under a variety of conditions, $\mathrm{Mgl}_{2}$ was found to be functional for this reaction, however producing low yields of product (Scheme 2). ${ }^{[7]}$ Interestingly the regioisomer isolated under these conditions was acetal $4 \mathbf{a}$ and not the aminal $3 \mathbf{a}$ as expected (compare schemes 1 and 2).

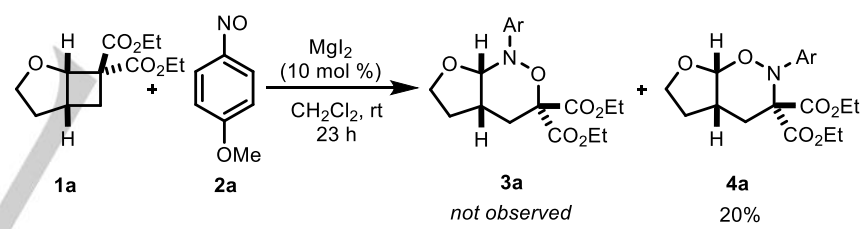

Scheme 2. Reaction of DA cyclobutane $1 \mathbf{a}$ with $\mathbf{2 a}$ under $\mathrm{Mgl}_{2}$ catalysis.

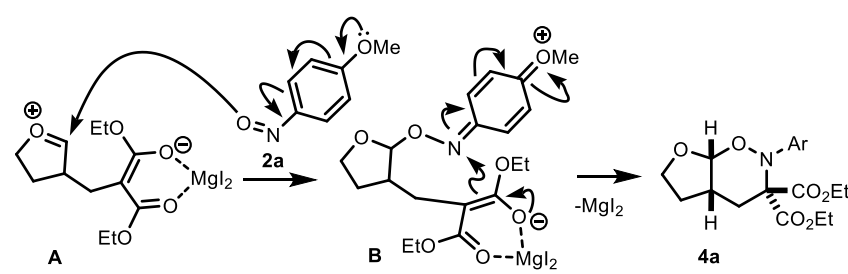

Scheme 3. Proposed mechanism for the formation of 4 a

This reversal in regioselectivity could be rationalized by the proposed mechanism (Scheme 3). The electron donating methoxy group on the aryl ring enhances the nucleophilicity of the nitroso oxygen, causing the oxygen to act as the nucleophile instead of nitrogen. Nucleophilic addition of the oxygen of the nitroso on the oxocarbenium ion $\mathbf{A}$ followed by cyclization via intermediate $\mathbf{B}$ would yield $\mathbf{4 a}$.

During the optimization of this reaction an interesting observation was made. ${ }^{[8]}$ When the reaction was left to stir for 2 days at room temperature or when $4 \mathrm{a}$ was treated with $50 \mathrm{~mol} \% \mathrm{Mgl}_{2}$ at room temperature overnight, pyrrolidine $\mathbf{5 a}$ was formed with trace amounts of lactone $\mathbf{6 a}$ (Scheme 4). 
<smiles>CCOC(=O)C1(C(=O)OCC)C[C@H]2CCO[C@H]2ON1c1ccc(OC)cc1</smiles>

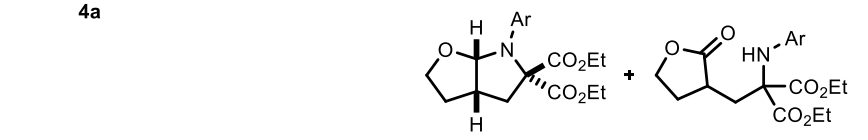

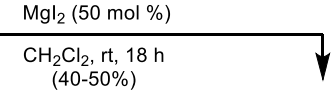

$5 a$ 6a trace<smiles>CCOC[C@]1([C@@H](CC)OCC)C[C@H]2CCO[C@H]21</smiles>

$1 \mathrm{a}$

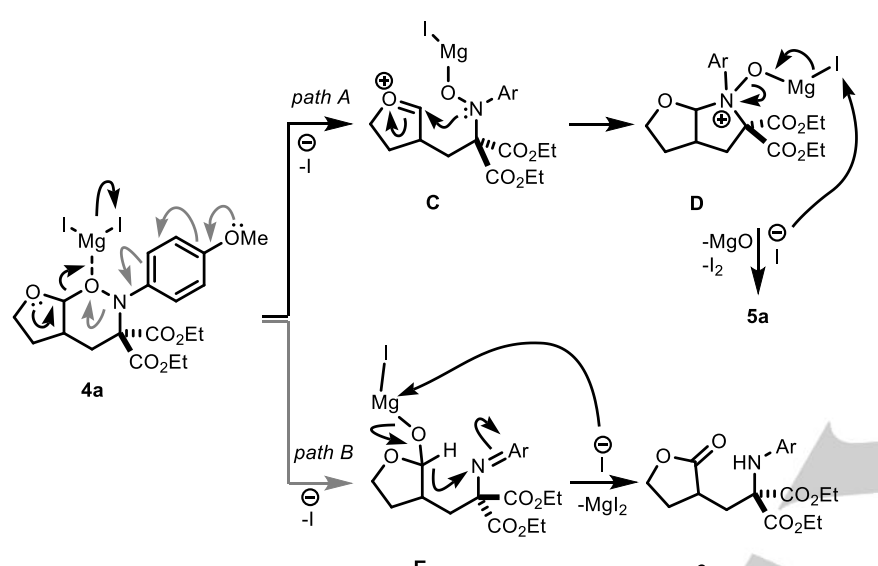

E

$6 a$

Scheme 5. Proposed mechanism for the formation of $\mathbf{5 a}$ and $\mathbf{6 a}$.

A postulated mechanism to explain this unique redox transformation is depicted in Scheme 5. Coordination of $\mathrm{Mgl}_{2}$ to the oxygen of tetrahydro-1,2-oxazine 4a polarizes both the $\mathrm{C}-\mathrm{O}$ and $\mathrm{N}-\mathrm{O}$ bonds indicated. Cleavage of the $\mathrm{C}-\mathrm{O}$ bond ( $P$ ath $A$ ) generates an oxocarbenium ion $\mathbf{C}$, which then undergoes nucleophilic attack by the pendant nitrogen, forming pyrrolidinium intermediate $\mathbf{D}$. Finally, the initially displaced iodide reacts with the $\mathrm{Mg}$ side chain on the nitrogen, resulting in $\mathrm{N}-\mathrm{O}$ bond reduction, and concomitantly producing $\mathrm{I}_{2}, \mathrm{MgO}$, and pyrrolidine $5 \mathrm{a}$. Lactone $6 \mathrm{a}$ can be formed via $\mathrm{N}-\mathrm{O}$ bond cleavage of $\mathbf{4 a}$ (Path $B$ ) leading to intermediate $\mathbf{E}$ followed by internal hydride migration.

Based on the proposed mechanism it is clear that stoichiometric $\mathrm{Mgl}_{2}$ was necessary for complete conversion of $\mathbf{4 a}$ to $\mathbf{5 a}$, but disappointingly the yields were further lowered with considerable decomposition of cyclobutane under such conditions.

With these interesting results we proceeded to investigate if other nitrosoarenes would follow a similar reaction manifold under $\mathrm{Mgl}_{2}$ promoted conditions. Thus, the reaction of electron-rich nitrosoarene $\mathbf{2 b}$ (which did not react under 10 or $20 \mathrm{~mol} \% \mathrm{Mgl}_{2}$ loadings) directly yielded pyrrolidine $\mathbf{5 b}$ (entry 2, Table 1), and isolation of the anticipated tetrahydro-1,2-oxazine $\mathbf{4 b}$ was not possible.

Table 1. Scope of the $\mathrm{Mgl}_{2}$ promoted cycloaddition.

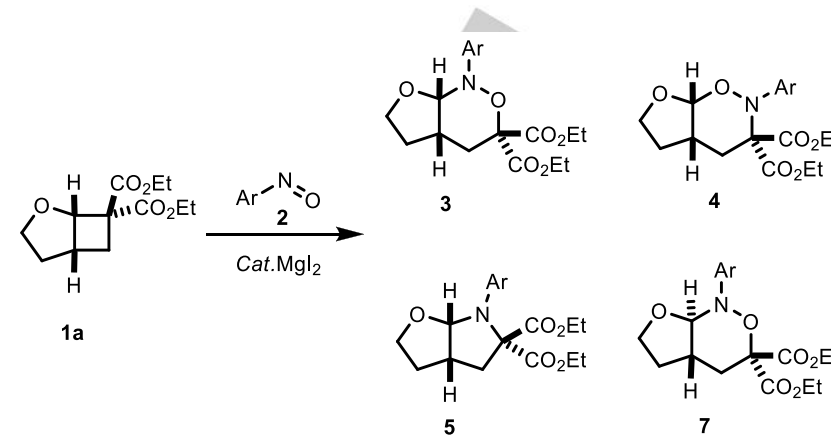

\begin{tabular}{clccc}
\hline Entry & \multicolumn{1}{c}{ Nitrosoarene } & Product & $\begin{array}{c}\mathrm{Mgl}_{2} \\
(\mathrm{~mol} \%)\end{array}$ & $\begin{array}{c}\text { Yield } \\
(\%)^{[b]}\end{array}$ \\
\hline 1 & $\mathbf{2 a ~ A r}=4-\mathrm{C}_{6} \mathrm{H}_{4} \mathrm{OMe}$ & $\mathbf{5 a}$ & 50 & 26 \\
2 & $\mathbf{2 b ~ A r}=4-\mathrm{C}_{6} \mathrm{H}_{4} \mathrm{~N}\left(\mathrm{CH}_{3}\right)_{2}$ & $\mathbf{5 b}$ & 50 & 22 \\
3 & $\mathbf{2} \mathbf{c ~ A r}=4-\mathrm{C}_{6} \mathrm{H}_{4} \mathrm{CN}$ & $\mathbf{4 c}, \mathbf{7 c}(2: 1)^{[\mathrm{a}]}$ & 10 & 35 \\
4 & $\mathbf{2 d ~ A r}=\mathrm{C}_{6} \mathrm{H}_{5}$ & $\mathbf{3 d}$ & 10 & 56 \\
5 & 2e Ar $=2$-pyridine & $\mathbf{4 e}$ & 50 & 28 \\
6 & 2f Ar $=$ N-BOC-5-nitrosoindole & $\mathbf{4 f}$ & 10 & 19 \\
\hline
\end{tabular}

[a] Ratio of 4:7 of isolated overall yields. [b] Isolated yields.

Interestingly, the substrate $\mathbf{2 c}$, which afforded aminal $\mathbf{3 c}$ as the major product under $\mathrm{Yb}(\mathrm{OTf})_{3}$ catalysis (Scheme 1), resulted in reversal of regioselectivity albeit in low yield (entry 3, Table 1). Also, the isolated aminal 7c was the trans-diastereomer (confirmed by single-crystal X-ray diffraction), ${ }^{[9]}$ rather than cis (3c, Scheme 1). ${ }^{[10]}$ Nitrosobenzene $\mathbf{2} \mathbf{d}$, which is electronically sandwiched between $\mathbf{2 a}$ and $\mathbf{2 c}$, produced aminal $\mathbf{3 d}$ as the exclusive product. The heteronitrosoarenes 2-nitrosopyridine $\mathbf{2 e}$ and $\mathrm{BOC}$ protected 5-nitrosoindole 2f, which did not react under $\mathrm{Yb}(\mathrm{OTf})_{3}$ conditions, provided exclusive acetal products (4e and $\mathbf{4 f}$ respectively) in low yields (entries 5 and 6 , Table 1).

Next other cyclobutanes were explored under both $\mathrm{Yb}(\mathrm{OTf})_{3}$ and $\mathrm{Mgl}_{2}$ reaction conditions (Table 2). The DA cyclobutane $\mathbf{1 b}$ gave a moderate yield with nitrosobenzene $\mathbf{2} \mathbf{d}$ under $\mathrm{Yb}(\mathrm{OTf})_{3}$ catalysis, while electron-rich nitrosoarenes $\mathbf{2} \mathbf{a}$ and $\mathbf{2} \mathbf{b}$ resulted in low yields of exclusive pyrrolidine products $9 a$ and $9 b$ respectively (entries 2 and 3 , Table 2). Crystals of $9 a$ were obtained and the X-ray structure unambiguously establishes the pyrrolidine product (Figure 1). ${ }^{[11]}$
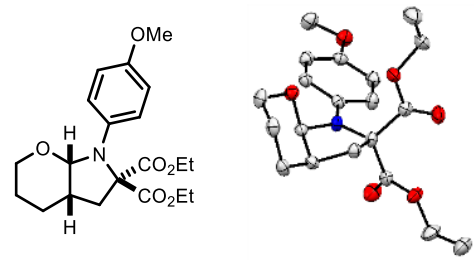

Figure 1. ORTEP structure of $9 a$ 
Table 2. Reaction scope of DA cyclobutanes in the cycloaddition.

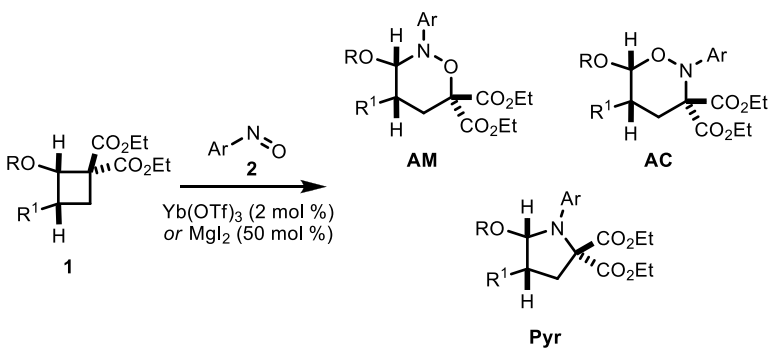

\begin{tabular}{llllc}
\hline Entry & Cyclobutane & Nitrosoarene & \multicolumn{2}{c}{ Product Yield [a] } \\
\cline { 3 - 4 } & & Yb(OTf $)_{3}$ & $\mathrm{Mgl}_{2}$ \\
\hline
\end{tabular}

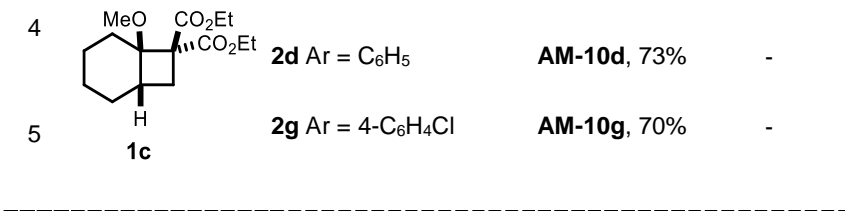

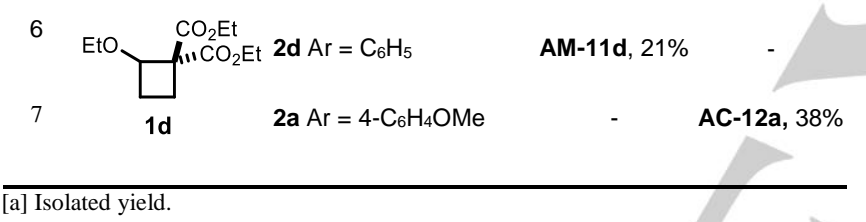

The DA cyclobutane 1c smoothly reacted with nitrosobenzene $\mathbf{2 d}$ and 1-chloro-4-nitrosobenzene $\mathbf{2 h}$ to furnish tetrahydro-1,2oxazines $\mathbf{1 0 d}$ and $\mathbf{1 0 g}$ in good yields (entries 4 and 5, Table 2). Disappointingly the ethoxy substituted DA cyclobutane $1 \mathrm{~d}$ gave a poor yield with nitrosobenzene $\mathbf{2 d}$, while electron-rich nitrosoarene $\mathbf{2 a}$ resulted in a low yield of acetal product $12 \mathbf{a}$ (entry 7 , Table 2 ).

Knowing that the acetal $\mathbf{4 a}$ could be converted into pyrrolidine 5 a via $\mathrm{Mgl}_{2}$ mediated reaction (Scheme 4), 12a was subjected 50 mol \% of $\mathrm{Mgl}_{2}$ in $\mathrm{CH}_{2} \mathrm{Cl}_{2}$ (Scheme 6 ). However, the reaction yielded ester 14a instead of pyrrolidine 13a.

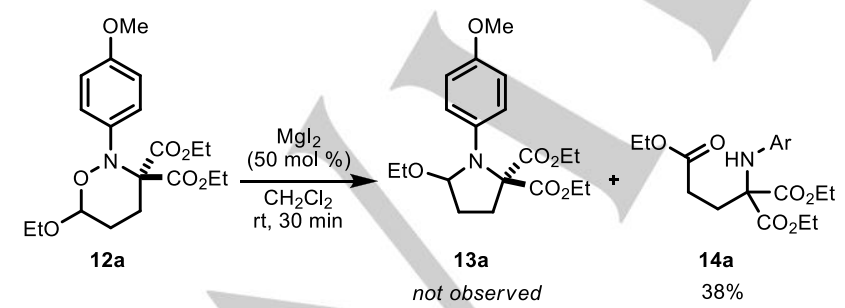

Scheme 6. Reaction of $12 \mathrm{a}$ under $\mathrm{Mgl}_{2}$ conditions.

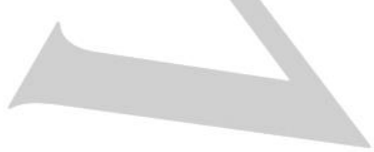

\section{Conclusions}

In conclusion, we have discovered the formation of unexpected pyrrolidine products in $\mathrm{Mgl}_{2}$ promoted cycloaddition reactions between DA cyclobutanes and electron-rich nitrosoarenes. The scope of $\mathrm{Yb}(\mathrm{OTf})_{3}$ catalyzed [4+2] cycloaddition between DA cyclobutanes and nitrosoarenes is extended to additional DA cyclobutanes. Furthermore a procedure to make pyrrolidines from tetrahydro-1,2oxazines was reported. The regiochemistry and stereochemistry of the unexpected pyrrolidine product $9 \mathbf{a}$ has been unambiguously assigned by single-crystal X-ray diffraction. Future work includes mechanistic studies to further efficiency of the process.

\section{Experimental Section}

\section{General experimental details:}

All reactions were run under argon atmosphere unless otherwise indicated. Flasks were oven dried and cooled in a desiccator prior to use. Solvents and reagents were purified by standard methods.[12 ] Dichloromethane was purified by passing the solvent through an activated alumina column. All other chemicals were of reagent quality and used as obtained from commercial sources unless otherwise noted. The progress of reactions were monitored by thin layer chromatography (TLC) performed on F254 silica gel plates. The plates were visualized by UV light $(254 \mathrm{~nm})$ or by staining with ceric ammonium molybdate (CAM). ${ }^{[13]}$ Column chromatography was performed with Silica Flash P60 60 Å silica gel from SiliCycle $\AA$ according to the Still method. ${ }^{[14]}$

The ${ }^{1} \mathrm{H}$ and ${ }^{13} \mathrm{C}$ NMR data were obtained on either 400 or $600 \mathrm{MHz}$ spectrometers. All spectra were obtained in deuterated chloroform and were referenced to the residual chloroform at $7.26 \mathrm{ppm}$ for ${ }^{1} \mathrm{H}$ spectra and the center peak of the triplet at $77.0 \mathrm{ppm}$ for ${ }^{13} \mathrm{C}$ spectra. When peak multiplicities are given, the following abbreviations are used: $\mathrm{s}$, singlet; $\mathrm{d}$, doublet; dd, doublet of doublets; ddd, doublet of doublet of doublets; $t$, triplet; q, quartet; quin, quintet; dt, doublet of triplets; dq, doublet of quartets; m, multiplet; br, broad; app, apparent. Scalar coupling was eliminated from nOe experiments by using acquisition delays of $500 \mathrm{~ms}$ Electron ionization mass spectra were obtained on a Finnigan MAT 8200 spectrometer at an ionizing voltage of $70 \mathrm{eV}$.

Nitrosoarenes, which are not commercially available, were prepared according to literature methods. ${ }^{[15]}$

Table 1. Catalyst screening for electron-rich nitrosoarenes.

\begin{tabular}{|c|c|c|c|c|}
\hline entry ${ }^{[a]}$ & catalyst & $\begin{array}{c}\mathrm{mol} \\
\%\end{array}$ & temperature, time & result ${ }^{[b]}$ \\
\hline 1 & $\mathrm{Yb}(\mathrm{OTf})_{3}$ & 50 & $\mathrm{rt}, 30 \mathrm{~min}$ & $19 \%[c]$ \\
\hline 2 & $\mathrm{Zn}(\mathrm{OTf})_{2}$ & 10 & $\mathrm{rt}, 20 \mathrm{~h}$ & $13 \%[c]$ \\
\hline 3 & $\mathrm{ZnCl}_{2}$ & 10 & $\mathrm{rt}, 6 \mathrm{~h}$ & $26 \%[c]$ \\
\hline 4 & $\mathrm{Zn}\left(\mathrm{NTf}_{2}\right)_{2}$ & 10 & $\mathrm{rt}, 2 \mathrm{~h}$ & $4 a-15 \%$ \\
\hline 5 & $\mathrm{AlCl}_{3}$ & 10 & $\mathrm{rt}, 23 \mathrm{~h}$ & $4 a-9 \%$ \\
\hline 6 & $\mathrm{AlBr}_{3}$ & 10 & $\mathrm{rt}, 72 \mathrm{~h}$ & no reaction ${ }^{[f]}$ \\
\hline 7 & $\mathrm{AlMe}_{3}$ & 20 & rt, $24 \mathrm{~h}$ & no reaction ${ }^{[f]}$ \\
\hline 8 & $\mathrm{MADNTf}_{2}$ & 100 & $\mathrm{rt}, 72 \mathrm{~h}$ & no reaction ${ }^{[\mathrm{f}]}$ \\
\hline 9 & $\mathrm{Et}_{2} \mathrm{AICl}$ & 20 & $0^{\circ} \mathrm{C}, 2 \mathrm{~h} ; \mathrm{rt}, 18 \mathrm{~h}$ & decomposition ${ }^{[\mathrm{e}}$ \\
\hline 10 & $\mathrm{Sc}(\mathrm{OTf})_{3}$ & 10 & $\mathrm{rt}, 30 \mathrm{~min}$ & decomposition ${ }^{[e]}$ \\
\hline 11 & Cul & 10 & rt, $52 \mathrm{~h}$ & no reaction ${ }^{[f]}$ \\
\hline 12 & $\mathrm{Cu}(\mathrm{OTf})_{2}$ & 10 & $\mathrm{rt}, 30 \mathrm{~min}$ & $4 a-14 \%$ \\
\hline 13 & $\mathrm{SnCl}_{2}$ & 10 & $\mathrm{rt}, 46 \mathrm{~h}$ & no reaction ${ }^{[\mathrm{f}]}$ \\
\hline 14 & $\mathrm{SnCl}_{4}$ & 10 & $0^{\circ} \mathrm{C}, 1 \mathrm{~h}$ & decomposition ${ }^{[\mathrm{e}]}$ \\
\hline 15 & $\mathrm{Sn}(\mathrm{OTf})_{2}$ & 10 & $\mathrm{rt}, 18 \mathrm{~h}$ & $4 a-10 \%$ \\
\hline
\end{tabular}




\begin{tabular}{|c|c|c|c|c|}
\hline 16 & $\mathrm{Bu}_{2} \mathrm{BOTf}$ & 10 & $0^{\circ} \mathrm{C}, 2 \mathrm{~h} ; \mathrm{rt}, 30 \mathrm{~min}$ & decomposition $^{[\mathrm{e}]}$ \\
\hline 17 & $\mathrm{BF}_{3} \cdot \mathrm{OEt}_{2}$ & 10 & $0^{\circ} \mathrm{C}, 4 \mathrm{~h} ; \mathrm{rt}, 18 \mathrm{~h}$ & decomposition ${ }^{[\mathrm{e}]}$ \\
\hline 18 & $\mathrm{AgOTf}$ & 10 & $\mathrm{rt}, 15 \mathrm{~min}$ & decomposition ${ }^{[\mathrm{e}]}$ \\
\hline 19 & $\mathrm{AgCl}$ & 10 & $\mathrm{rt}, 18 \mathrm{~h}$ & decomposition ${ }^{[\mathrm{e}]}$ \\
\hline 20 & $\mathrm{ZrCl}_{4}$ & 10 & $0^{\circ} \mathrm{C}, 3 \mathrm{~h} ; \mathrm{rt}, 18 \mathrm{~h}$ & decomposition ${ }^{[\mathrm{e}]}$ \\
\hline 21 & $\mathrm{TiCl}_{4}$ & 10 & $0^{\circ} \mathrm{C}, 3 \mathrm{~h} ; \mathrm{rt}, 18 \mathrm{~h}$ & decomposition ${ }^{[e]}$ \\
\hline 22 & $\ln \left(\mathrm{NTf}_{2}\right)_{3}$ & 10 & $\mathrm{rt}, 30 \mathrm{~min}$ & $4 a-20 \%$ \\
\hline 23 & $\ln (\mathrm{OTf})_{3}$ & 10 & $\mathrm{rt}, 3.5 \mathrm{~h}$ & $4 a-13 \%$ \\
\hline 24 & $\mathrm{MgCl}_{2}$ & 10 & $\mathrm{rt}, 100 \mathrm{~h}$ & no reaction ${ }^{[f]}$ \\
\hline 25 & $\mathrm{MgBr}_{2}$ & 10 & $\mathrm{rt}, 120 \mathrm{~h}$ & $4 a-4 \%$ \\
\hline 26 & $\mathrm{Mg}\left(\mathrm{ClO}_{4}\right)_{2}$ & 10 & $0^{\circ} \mathrm{C}, 4 \mathrm{~h} ; \mathrm{rt}, 2 \mathrm{~h}$ & decomposition ${ }^{[\mathrm{e}]}$ \\
\hline 27 & $\mathrm{Mgl}_{2}$ & 10 & $\mathrm{rt}, 23 \mathrm{~h}$ & $4 a-20 \%$ \\
\hline 28 & $\mathrm{Mgl}_{2}$ & 5 & $\mathrm{rt}, 24 \mathrm{~h}$ & $4 a-17 \%$ \\
\hline 29 & $\mathrm{Mgl}_{2}$ & 10 & $0^{\circ} \mathrm{C}, 40 \mathrm{~h}$ & $4 a-30 \%$ \\
\hline 30 & $\mathrm{Mgl}_{2}$ & 10 & $-20^{\circ} \mathrm{C}, 72 \mathrm{~h}$ & $4 a-20 \%$ \\
\hline 31 & $\mathrm{Mgl}_{2}$ & 50 & $0^{\circ} \mathrm{C}, 15 \mathrm{~min}$ & $4 a-26 \%$ \\
\hline 32 & $\mathrm{Mgl}_{2}$ & 50 & $\mathrm{rt}, 18 \mathrm{~h}$ & $\begin{array}{l}\mathbf{4 a - 1 3 \% , 5 a -} \\
13 \%[\mathrm{~g}]\end{array}$ \\
\hline 33 & $\mathrm{Mgl}_{2}$ & 50 & $\mathrm{rt}, 48 \mathrm{~h}$ & $5 a-22 \%{ }^{[g]}$ \\
\hline 34 & $\mathrm{Mgl}_{2}$ & 10 & $\begin{array}{l}40{ }^{\circ} \mathrm{C}, \quad 45 \min ^{[d]} ; \\
100{ }^{\circ} \mathrm{C}, 30 \mathrm{~min}^{[\mathrm{d}]}\end{array}$ & $\begin{array}{l}\mathbf{4 a}-9 \% \\
11 \%[g]\end{array}$ \\
\hline
\end{tabular}

[a] Typical reaction conditions: To a solution of Lewis acid in $\mathrm{CH}_{2} \mathrm{Cl}_{2}(3 \mathrm{~mL})$ at specified temperature, was added nitrosoarene $2 \mathrm{a}(0.3 \mathrm{mmol})$ followed by cyclobutane 1a $(0.36 \mathrm{mmol})$. Reactions were monitored until nitrosoarene $\mathbf{2 a}$ was consumed by TLC. ${ }^{[b]}$ Isolated yields. ${ }^{[c]} 1: 1$ mixture $4 a$ and another unknown compound by ${ }^{1} \mathrm{H}$ NMR. ${ }^{[\mathrm{d}]}$ Microwave irradiation. ${ }^{[\mathrm{e}]}$ Cyclobutane 1a consumed. [f] Cyclobutane 1a recovered with some decomposition based on TLC and/or crude NMR. ${ }^{[g]}$ Trace amount of $6 \mathbf{a}$ was also isolated.

\section{General $\mathrm{Yb}(\mathrm{OTf})_{3}$ catalyzed cycloaddition procedure}

To a stirred solution of $\mathrm{Yb}(\mathrm{OTf})_{3}\left(4 \mathrm{mg}, 0.006 \mathrm{mmol}, 2 \mathrm{~mol} \%\right.$ ) in $\mathrm{CH}_{2} \mathrm{Cl}_{2}$ (3 $\mathrm{mL}$ ) at room temperature was added nitrosoarene $(0.30 \mathrm{mmol}, 1.0$ equiv) followed by cyclobutane $(0.36 \mathrm{mmol}, 1.2$ equiv). After complete consumption of the nitrosoarene (as indicated by TLC) the reaction mixture was layered directly onto a silica gel column and purified by flash chromatography (0-25\% EtOAc/hexanes).

\section{General $\mathrm{Mgl}_{2}$ catalyzed cycloaddition procedure}

To a stirred solution of $\mathrm{Mgl}_{2}(41.5 \mathrm{mg}, 0.15 \mathrm{mmol}, 50 \mathrm{~mol} \%)$ in $\mathrm{CH}_{2} \mathrm{Cl}_{2}$ (3 $\mathrm{mL})$ at $0{ }^{\circ} \mathrm{C}$ was added nitrosoarene $(0.30 \mathrm{mmol}, 1.0$ equiv) followed by cyclobutane ( $0.36 \mathrm{mmol}, 1.2$ equiv). After complete consumption of the nitrosoarene (as indicated by TLC) the reaction mixture was layered directly onto a silica gel column and purified by flash chromatography (0$25 \%$ EtOAc/hexanes)

Compound 4a: The title compound was prepared according to the general $\mathrm{Mgl}_{2}$ catalyzed cycloaddition procedure at $0{ }^{\circ} \mathrm{C}$ for 15 minutes to afford a pale yellow paste $(30 \mathrm{mg}, 26 \%) ; \mathrm{R}_{f} 0.41$ (1:1 hexanes /EtOAc); ${ }^{1} \mathrm{H}$ NMR $\left(600 \mathrm{MHz}, \mathrm{CDCl}_{3}\right) \delta 7.24-7.26(\mathrm{~m}, 2 \mathrm{H}), 6.75-6.77(\mathrm{~m}, 2 \mathrm{H}), 5.55(\mathrm{~d}, J$ $=5.3 \mathrm{~Hz}, 1 \mathrm{H}), 4.20-4.24(\mathrm{~m}, 1 \mathrm{H}), 4.15-4.19(\mathrm{~m}, 1 \mathrm{H}), 4.07-4.13(\mathrm{~m}$, $2 \mathrm{H}), 4.01-4.06(\mathrm{~m}, 1 \mathrm{H}), 3.96(\mathrm{td}, J=8.1,5.0 \mathrm{~Hz}, 1 \mathrm{H}), 3.76(\mathrm{~s}, 3 \mathrm{H})$, $2.61-2.72(\mathrm{~m}, 2 \mathrm{H}), 2.50-2.57(\mathrm{~m}, 1 \mathrm{H}), 2.01-2.09(\mathrm{~m}, 1 \mathrm{H}), 1.95(\mathrm{dq}$, $J=12.2,6.3 \mathrm{~Hz}, 1 \mathrm{H}$ ), 1.14 (t, $J=7.0 \mathrm{~Hz}, 3 \mathrm{H}$ ), 1.10 (t, $J=7.0 \mathrm{~Hz}, 3 \mathrm{H}$ ); ${ }^{13} \mathrm{C}$ NMR $\left(101 \mathrm{MHz}, \mathrm{CDCl}_{3}\right) \delta 169.0,168.8,156.6,140.7,122.7,113.0$, 104.5, 74.3, 68.8, 61.9, 61.6, 55.4, 35.1, 32.3, 28.9, 13.8, 13.8; HRMS m/z 379.1635 (calcd for $\mathrm{C}_{19} \mathrm{H}_{25} \mathrm{NO}_{7}, 379.1631$ ).

Compound 5a: To a stirred solution of $\mathrm{Mgl}_{2}(44 \mathrm{mg}, 0.16 \mathrm{mmol})$ in $10 \mathrm{~mL}$ $\mathrm{CH}_{2} \mathrm{Cl}_{2}$ at room temperature was added $4 \mathrm{a}(120 \mathrm{mg}, 0.32 \mathrm{mmol})$. The reaction mixture was stirred for about 18 hours at room temperature (complete consumption of starting material by TLC) then the reaction mixture was layered directly onto a silica gel column and purified by flash chromatography (0-25\% EtOAc/hexanes); $\mathrm{R}_{f} 0.37$ (1:1 hexanes /EtOAc); ${ }^{1} \mathrm{H}$ NMR $\left(600 \mathrm{MHz}, \mathrm{CDCl}_{3}\right) \delta 6.90-6.94(\mathrm{~m}, 2 \mathrm{H}), 6.75-6.78(\mathrm{~m}, 2 \mathrm{H})$, $5.62(\mathrm{~d}, J=5.9 \mathrm{~Hz}, 1 \mathrm{H}), 4.15-4.25(\mathrm{~m}, 4 \mathrm{H}), 3.91-4.00(\mathrm{~m}, 2 \mathrm{H}), 3.74$ (s, $3 \mathrm{H}), 3.00-3.07(\mathrm{~m}, 1 \mathrm{H}), 2.70(\mathrm{dd}, J=12.9,8.8 \mathrm{~Hz}, 1 \mathrm{H}), 2.39(\mathrm{dd}, J$ $=13.5,8.8 \mathrm{~Hz}, 1 \mathrm{H}), 2.00-2.08(\mathrm{~m}, 1 \mathrm{H}), 1.72(\mathrm{dd}, J=12.6,5.0 \mathrm{~Hz}, 1 \mathrm{H})$, $1.21(\mathrm{t}, J=7.3 \mathrm{~Hz}, 3 \mathrm{H}), 1.18(\mathrm{t}, J=7.3 \mathrm{~Hz}, 3 \mathrm{H}) ;{ }^{13} \mathrm{C} \mathrm{NMR}(101 \mathrm{MHz}$, $\left.\mathrm{CDCl}_{3}\right) \delta 170.7,169.9,153.4,138.3,117.6,113.9,98.6,74.8,65.5,61.8$, $61.8,55.5,40.6,40.1,32.0,14.0,14.0$; HRMS $\mathrm{m} / \mathrm{z} 363.1692$ (calcd for $\left.\mathrm{C}_{19} \mathrm{H}_{25} \mathrm{NO}_{6}, 363.1682\right)$.

Compound 6a: The title compound was isolated in trace amount along with $5 \mathrm{a}$ as pale brown oil; $\mathrm{R}_{f} 0.24$ (70\% hexanes /EtOAc); ${ }^{1} \mathrm{H}$ NMR $(600$ $\left.\mathrm{MHz}, \mathrm{CDCl}_{3}\right) \delta 6.75-6.72(\mathrm{~m}, 2 \mathrm{H}), 6.65-6.62(\mathrm{~m}, 2 \mathrm{H}), 4.89(\mathrm{~s}, 1 \mathrm{H})$, $4.29-4.15(\mathrm{~m}, 5 \mathrm{H}), 4.06$ (ddd, $J=11.2,9.1,6.2 \mathrm{~Hz}, 1 \mathrm{H}), 3.72(\mathrm{~s}, 3 \mathrm{H})$, 3.09 (dd, $J=15.3,3.5 \mathrm{~Hz}, 1 \mathrm{H}), 2.67-2.60(\mathrm{~m}, 1 \mathrm{H}), 2.44(\mathrm{dd}, J=15.3$ $10.0 \mathrm{~Hz}, 1 \mathrm{H}), 2.34-2.27(\mathrm{~m}, 1 \mathrm{H}), 1.78(\mathrm{qd}, J=11.7,8.8 \mathrm{~Hz}, 1 \mathrm{H}), 1.21$ $-1.16(\mathrm{~m}, 6 \mathrm{H}) ;{ }^{13} \mathrm{C}$ NMR $\left(101 \mathrm{MHz}, \mathrm{CDCl}_{3}\right) \delta 178.8,169.9,169.2,153.3$ $137.5,116.9,114.7,67.9,66.4,62.5,62.5,55.5,35.2,33.7,29.7,13.9$; HRMS $m / z 379.1639$ (calcd for $\mathrm{C}_{19} \mathrm{H}_{25} \mathrm{NO}_{7}, 379.1631$ ).

Compound 5b: The title compound was prepared according to the general $\mathrm{Mgl}_{2}$ catalyzed cycloaddition procedure at room temperature for 4 hours to afford a yellow colored oil ( $25 \mathrm{mg}, 22 \%) ; \mathrm{R}_{f} 0.33$ (60\% hexanes /EtOAc); ${ }^{1} \mathrm{H} \mathrm{NMR}\left(600 \mathrm{MHz}, \mathrm{CDCl}_{3}\right) \delta 6.90-6.94(\mathrm{~m}, 2 \mathrm{H}), 6.66-6.70(\mathrm{~m}, 2 \mathrm{H})$, $5.62(\mathrm{~d}, J=5.9 \mathrm{~Hz}, 1 \mathrm{H}), 4.13-4.24(\mathrm{~m}, 4 \mathrm{H}), 3.91-3.98(\mathrm{~m}, 2 \mathrm{H}), 2.99$ $-3.06(\mathrm{~m}, 1 \mathrm{H}), 2.82(\mathrm{~s}, 6 \mathrm{H}), 2.68(\mathrm{dd}, J=13.5,8.8 \mathrm{~Hz}, 1 \mathrm{H}), 2.37(\mathrm{dd}, J$ $=13.2,8.5 \mathrm{~Hz}, 1 \mathrm{H}), 1.98-2.06(\mathrm{~m}, 1 \mathrm{H}), 1.70(\mathrm{dd}, J=12.6,4.4 \mathrm{~Hz}, 1 \mathrm{H})$, $1.15-1.21(\mathrm{~m}, 6 \mathrm{H}) ;{ }^{13} \mathrm{C}$ NMR $\left(101 \mathrm{MHz}, \mathrm{CDCl}_{3}\right) \delta 170.9,170.0,145.3$ 136.0, 118.2, 114.3, 98.7, 74.8, 65.4, 61.7, 61.6, 41.7, 40.5, 40.0, 32.2, 14.0, 14.0; HRMS $m / z$ 376.2044 (calcd for $\mathrm{C}_{20} \mathrm{H}_{28} \mathrm{~N}_{2} \mathrm{O}_{5}, 376.1998$ ).

Compound 7c:[9] The title compound was prepared along with 4c according to the general $\mathrm{Mgl}_{2}(10 \mathrm{~mol} \%)$ catalyzed cycloaddition procedure at room temperature for 2 hours to afford a pale brown solid (15 $\mathrm{mg}, 13 \%) ; \mathrm{R}_{f} 0.44\left(70 \%\right.$ hexanes /EtOAc); ${ }^{1} \mathrm{H}$ NMR $\left(600 \mathrm{MHz}, \mathrm{CDCl}_{3}\right) \delta$ $7.60-7.63(\mathrm{~m}, 2 \mathrm{H}), 7.54-7.59(\mathrm{~m}, 2 \mathrm{H}), 4.22-4.34(\mathrm{~m}, 5 \mathrm{H}), 4.06-$ $4.15(\mathrm{~m}, 2 \mathrm{H}), 2.98(\mathrm{dd}, J=12.9,3.5 \mathrm{~Hz}, 1 \mathrm{H}), 2.24-2.32(\mathrm{~m}, 1 \mathrm{H}), 2.19$ $-2.24(\mathrm{~m}, 1 \mathrm{H}), 1.87(\mathrm{t}, J=12.9 \mathrm{~Hz}, 1 \mathrm{H}), 1.70-1.79(\mathrm{~m}, 1 \mathrm{H}), 1.30(\mathrm{t}, J$ $=7.0 \mathrm{~Hz}, 3 \mathrm{H}), 1.24(\mathrm{t}, J=7.0 \mathrm{~Hz}, 3 \mathrm{H}) ;{ }^{13} \mathrm{C} \mathrm{NMR}\left(101 \mathrm{MHz}, \mathrm{CDCl}_{3}\right) \delta$ 167.0, 166.2, 151.9, 132.5, 119.4, 119.2, 107.2, 94.5, 84.0, 67.7, 62.6, 62.3, 41.4, 34.1, 27.9, 14.0, 14.0; HRMS $\mathrm{m} / \mathrm{z} 374.1480$ (calcd for $\left.\mathrm{C}_{19} \mathrm{H}_{22} \mathrm{~N}_{2} \mathrm{O}_{6}, 374.1478\right)$

Compound 4e: The title compound was prepared according to the general $\mathrm{Mgl}_{2}$ catalyzed cycloaddition procedure at room temperature for 2 hours to afford a yellow syrup $(30 \mathrm{mg}, 28 \%) ; \mathrm{R}_{f} 0.34\left(70 \%\right.$ hexanes /EtOAc); ${ }^{1} \mathrm{H}$ NMR $\left(600 \mathrm{MHz}, \mathrm{CDCl}_{3}\right) \delta 8.01-8.04(\mathrm{~m}, 1 \mathrm{H}), 7.55-7.60(\mathrm{~m}, 1 \mathrm{H}), 7.19$ $-7.23(\mathrm{~m}, 1 \mathrm{H}), 6.75-6.79(\mathrm{~m}, 1 \mathrm{H}), 5.58(\mathrm{~d}, J=4.7 \mathrm{~Hz}, 1 \mathrm{H}), 4.22-4.32$ $(\mathrm{m}, 2 \mathrm{H}), 4.19$ (q, $J=7.0 \mathrm{~Hz}, 1 \mathrm{H}), 4.10-4.17(\mathrm{~m}, 1 \mathrm{H}), 3.93(\mathrm{q}, J=8.0$ $\mathrm{Hz}, 1 \mathrm{H}), 2.68-2.77(\mathrm{~m}, 2 \mathrm{H}), 2.53-2.60(\mathrm{~m}, 1 \mathrm{H}), 1.98-2.10(\mathrm{~m}, 2 \mathrm{H})$ $1.22-1.27(\mathrm{~m}, 3 \mathrm{H}), 1.13-1.17(\mathrm{~m}, 3 \mathrm{H}) ;{ }^{13} \mathrm{C} \mathrm{NMR}\left(101 \mathrm{MHz}, \mathrm{CDCl}_{3}\right) \delta$ 168.9, 168.7, 159.3, 145.6, 137.5, 116.7, 109.8, 104.1, 69.9, 69.1, 62.0, 61.6, 36.1, 31.8, 27.6, 13.8, 13.7; HRMS $\mathrm{m} / \mathrm{z} 350.1470$ (calcd for $\left.\mathrm{C}_{17} \mathrm{H}_{22} \mathrm{~N}_{2} \mathrm{O}_{6}, 350.1478\right)$.

Compound 4f: The title compound was prepared according to the general $\mathrm{Mgl}_{2}(10 \mathrm{~mol} \%$ ) catalyzed cycloaddition procedure at room temperature for 4 hours to afford a pale yellow oil $(28 \mathrm{mg}, 19 \%) ; \mathrm{R}_{f} 0.29(70 \%$ hexanes /EtOAc); ${ }^{1} \mathrm{H}$ NMR $\left(400 \mathrm{MHz}, \mathrm{CDCl}_{3}\right) \delta 7.94(\mathrm{~d}, J=8.6 \mathrm{~Hz}, 1 \mathrm{H}), 7.52(\mathrm{~d}, J$ $=3.5 \mathrm{~Hz}, 1 \mathrm{H}), 7.50(\mathrm{~d}, J=2.3 \mathrm{~Hz}, 1 \mathrm{H}), 7.26(\mathrm{dd}, J=9.0,2.3 \mathrm{~Hz}, 1 \mathrm{H})$, $6.47(\mathrm{~d}, J=3.5 \mathrm{~Hz}, 1 \mathrm{H}), 5.60(\mathrm{~d}, J=5.5 \mathrm{~Hz}, 1 \mathrm{H}), 3.94-4.28(\mathrm{~m}, 6 \mathrm{H})$ $2.64-2.77(\mathrm{~m}, 2 \mathrm{H}), 2.51-2.62(\mathrm{~m}, 1 \mathrm{H}), 2.02-2.13(\mathrm{~m}, 1 \mathrm{H}), 1.92-$ $2.02(\mathrm{~m}, 1 \mathrm{H}), 1.65(\mathrm{~s}, 9 \mathrm{H}), 1.12(\mathrm{t}, J=7.0 \mathrm{~Hz}, 3 \mathrm{H}), 1.04(\mathrm{t}, J=7.0 \mathrm{~Hz}, 3$ $\mathrm{H}) ;{ }^{13} \mathrm{C}$ NMR $\left(101 \mathrm{MHz}, \mathrm{CDCl}_{3}\right) \delta 169.0,168.9,142.9,130.1,126.1,118.0$, $114.1,112.9,107.5,104.5,83.4,74.4,68.8,62.0,61.6,35.3,32.6,29.0$ 28.2, 13.8, 13.7; HRMS $\mathrm{m} / \mathrm{z} 488.2153$ (calcd for $\mathrm{C}_{25} \mathrm{H}_{32} \mathrm{~N}_{2} \mathrm{O}_{8}, 488.2159$ ). 
Compound 8d: The title compound was prepared according to the general $\mathrm{Yb}(\mathrm{OTf})_{3}$ catalyzed cycloaddition procedure for 4 hours to afford a pale yellow solid (50 mg, 45\%); $\mathrm{R}_{f} 0.25$ (70\% hexanes /EtOAc); ${ }^{1} \mathrm{H}$ NMR (600 $\left.\mathrm{MHz}, \mathrm{CDCl}_{3}\right) \delta 7.22-7.31(\mathrm{~m}, 4 \mathrm{H}), 7.00(\mathrm{t}, \mathrm{J}=7.0 \mathrm{~Hz}, 1 \mathrm{H}), 4.88(\mathrm{~d}, J=$ $2.9 \mathrm{~Hz}, 1 \mathrm{H}), 4.26-4.33(\mathrm{~m}, 2 \mathrm{H}), 4.14-4.26(\mathrm{~m}, 2 \mathrm{H}), 4.04-4.10(\mathrm{~m}, 1$ $\mathrm{H}), 3.50$ (td, $J=11.7,1.8 \mathrm{~Hz}, 1 \mathrm{H}), 2.42-2.49(\mathrm{~m}, 1 \mathrm{H}), 2.22-2.35(\mathrm{~m}, 2$ $\mathrm{H}), 1.87-1.95(\mathrm{~m}, 1 \mathrm{H}), 1.76-1.86(\mathrm{~m}, 2 \mathrm{H}), 1.31(\mathrm{t}, J=7.0 \mathrm{~Hz}, 3 \mathrm{H})$, $1.16(\mathrm{t}, J=7.0 \mathrm{~Hz}, 3 \mathrm{H}) ;{ }^{13} \mathrm{C}$ NMR $\left(101 \mathrm{MHz}^{\mathrm{C}} \mathrm{CDCl}_{3}\right) \delta 168.0,166.9,146.6$, $128.5,122.9,117.4,84.4,67.9,62.1,61.8,29.9,28.5,27.5,20.1,14.1$, 14.0; HRMS $m / z 363.1688$ (calcd for $\mathrm{C}_{19} \mathrm{H}_{25} \mathrm{NO}_{6}, 363.1682$ ).

Compound 9a: ${ }^{[11]}$ The title compound was prepared according to the general $\mathrm{Mgl}_{2}$ catalyzed cycloaddition procedure at $0{ }^{\circ} \mathrm{C}$ for 15 minutes to afford a pale brown solid (40 mg, 35\%); $\mathrm{R}_{f} 0.26$ (70\% hexanes /EtOAc); ${ }^{1} \mathrm{H}$ $\operatorname{NMR}\left(600 \mathrm{MHz}, \mathrm{CDCl}_{3}\right) \delta 6.90-6.94(\mathrm{~m}, 2 \mathrm{H}), 6.72-6.77(\mathrm{~m}, 2 \mathrm{H}), 5.03$ $(\mathrm{d}, J=3.5 \mathrm{~Hz}, 1 \mathrm{H}), 4.15-4.25(\mathrm{~m}, 4 \mathrm{H}), 3.90-3.96(\mathrm{~m}, 1 \mathrm{H}), 3.73$ (s, 3 H), 3.44 (td, $J=11.3,2.1 \mathrm{~Hz}, 1 \mathrm{H}), 2.84(\mathrm{t}, J=12.3 \mathrm{~Hz}, 1 \mathrm{H}), 2.43$ (dd, $J=$ $12.0,6.8 \mathrm{~Hz}, 1 \mathrm{H}), 2.33-2.40(\mathrm{~m}, 1 \mathrm{H}), 1.85-1.94(\mathrm{~m}, 1 \mathrm{H}), 1.69-1.80$ (m, $2 \mathrm{H}), 1.38-1.44(\mathrm{~m}, 1 \mathrm{H}), 1.22(\mathrm{t}, J=7.0 \mathrm{~Hz}, 3 \mathrm{H}), 1.16(\mathrm{t}, J=7.0 \mathrm{~Hz}$, $3 \mathrm{H}) ;{ }^{13} \mathrm{C} \mathrm{NMR}\left(101 \mathrm{MHz}, \mathrm{CDCl}_{3}\right) \delta 171.1,153.3,137.8,118.0,113.8,90.0$, 74.0, 63.9, 61.7, 61.7, 55.5, 38.2, 35.1, 23.7, 20.7, 14.0, 13.9; HRMS m/z 377.1837 (calcd for $\mathrm{C}_{20} \mathrm{H}_{27} \mathrm{NO}_{6}, 377.1838$ ).

Compound 9b: The title compound was prepared according to the general $\mathrm{Mgl}_{2}$ catalyzed cycloaddition procedure at $0^{\circ} \mathrm{C}$ for 1 hour, followed by 1 hour at room temperature to afford a yellow oil (22 mg, 19\%); $\mathrm{R}_{f} 0.43(50 \%$ hexanes /EtOAc); ${ }^{1} \mathrm{H}$ NMR $\left(600 \mathrm{MHz}, \mathrm{CDCl}_{3}\right) \delta 6.92-6.96(\mathrm{~m}, 2 \mathrm{H}), 6.65$ $-6.68(\mathrm{~m}, 2 \mathrm{H}), 5.01(\mathrm{~d}, J=4.1 \mathrm{~Hz}, 1 \mathrm{H}), 4.15-4.21(\mathrm{~m}, 4 \mathrm{H}), 3.92(\mathrm{~d}, J$ $=11.2 \mathrm{~Hz}, 1 \mathrm{H}), 3.42(\mathrm{td}, J=11.0,2.1 \mathrm{~Hz}, 1 \mathrm{H}), 2.82(\mathrm{~s}, 6 \mathrm{H}), 2.39-2.44$ $(\mathrm{m}, 1 \mathrm{H}), 2.33-2.39(\mathrm{~m}, 1 \mathrm{H}), 1.83-1.93(\mathrm{~m}, 1 \mathrm{H}), 1.68-1.80(\mathrm{~m}, 2 \mathrm{H})$, $1.37-1.43(\mathrm{~m}, 1 \mathrm{H}), 1.24-1.30(\mathrm{~m}, 1 \mathrm{H}), 1.21(\mathrm{t}, J=7.3 \mathrm{~Hz}, 3 \mathrm{H}), 1.15(\mathrm{t}$, $J=7.0 \mathrm{~Hz}, 3 \mathrm{H}) ;{ }^{13} \mathrm{C}$ NMR $\left(101 \mathrm{MHz}, \mathrm{CDCl}_{3}\right) \delta 171.4,170.4,145.5,135.5$ $118.9,114.3,90.2,74.1,63.8,61.6,61.5,41.8,38.1,35.2,23.8,20.8,14.0$ 13.9; HRMS $m / z 390.2167$ (calcd for $\mathrm{C}_{21} \mathrm{H}_{30} \mathrm{~N}_{2} \mathrm{O}_{5}, 390.2155$ ).

Compound 10d: The title compound was prepared according to the general $\mathrm{Yb}(\mathrm{OTf})_{3}$ catalyzed cycloaddition procedure for 15 minutes to afford a pale yellow oil (85 mg, 73\%); $\mathrm{R}_{f} 0.59$ (70\% hexanes /EtOAc); ${ }^{1} \mathrm{H}$ $\operatorname{NMR}\left(600 \mathrm{MHz}, \mathrm{CDCl}_{3}\right) \delta 7.18$ - $7.24(\mathrm{~m}, 4 \mathrm{H}), 6.98-7.01(\mathrm{~m}, 1 \mathrm{H}), 4.11$ $4.22(\mathrm{~m}, 4 \mathrm{H}), 3.13$ (s, $3 \mathrm{H}), 2.60$ (t, $J=12.6 \mathrm{~Hz}, 1 \mathrm{H}), 2.14$ (dd, $J=12.6$, $3.8 \mathrm{~Hz}, 1 \mathrm{H}), 1.95-2.00(\mathrm{~m}, 1 \mathrm{H}), 1.66-1.75(\mathrm{~m}, 2 \mathrm{H}), 1.60-1.65(\mathrm{~m}, 1$ $\mathrm{H}), 1.43-1.48(\mathrm{~m}, 2 \mathrm{H}), 1.21-1.35(\mathrm{~m}, 3 \mathrm{H}), 1.12-1.18(\mathrm{~m}, 6 \mathrm{H}) ;{ }^{13} \mathrm{C}$ NMR $\left(101 \mathrm{MHz}, \mathrm{CDCl}_{3}\right) \delta 168.8,167.9,148.8,127.6,123.3,120.5,101.4,76.3$, 62.1, 61.0, 48.1, 40.6, 33.8, 29.3, 27.6, 25.5, 22.5, 13.9, 13.7; HRMS m/z 391.1987 (calcd for $\mathrm{C}_{21} \mathrm{H}_{29} \mathrm{NO}_{6}, 391.1995$ ).

Compound 10g: The title compound was prepared according to the general $\mathrm{Yb}(\mathrm{OTf})_{3}$ catalyzed cycloaddition procedure for 45 minutes to afford a pale yellow oil (90 mg, 70\%); $R_{f} 0.65$ (70\% hexanes /EtOAc); ${ }^{1} \mathrm{H}$ $\operatorname{NMR}\left(600 \mathrm{MHz}, \mathrm{CDCl}_{3}\right) \delta 7.16(\mathrm{~s}, 4 \mathrm{H}), 4.11-4.23(\mathrm{~m}, 4 \mathrm{H}), 3.10(\mathrm{~s}, 3 \mathrm{H})$, 2.58 (t, $J=12.6 \mathrm{~Hz}, 1 \mathrm{H}), 2.12$ (dd, $J=12.6,3.8 \mathrm{~Hz}), 1.93-1.99(\mathrm{~m}, 1 \mathrm{H})$, $1.60-1.75(\mathrm{~m}, 3 \mathrm{H}), 1.42-1.52(\mathrm{~m}, 3 \mathrm{H}), 1.26-1.36(\mathrm{~m}, 1 \mathrm{H}), 1.19-1.26$ $(\mathrm{m}, 1 \mathrm{H}), 1.14-1.19(\mathrm{~m}, 6 \mathrm{H}) ;{ }^{13} \mathrm{C} \mathrm{NMR}\left(101 \mathrm{MHz}, \mathrm{CDCl}_{3}\right) \delta 168.6,167.8$,

[1] For reviews, see; (a) K. V. Gothelf and K. A. Jørgensen, Chem. Rev 1998, 98, 863; (b) S. Kobayashi and K. A. Jørgensen, Cycloaddition Reactions in Organic Synthesis, Wiley-VCH Verlag $\mathrm{GmbH}$, Weinheim, 2001.

[2] For reviews, see; (a) A. Padwa and W. H. Pearson, Synthetic Applications of 1,3-Dipolar Cycloaddition Chemistry Toward Heterocycles and Natural Products, John Wiley \& Sons, Inc., New York, 2003; (b) L. M. Stanley and M. P. Sibi, Chem. Rev. 2008, 108, 2887.
147.5, 127.6, 122.0, 101.6, 76.4, 62.2, 61.2, 48.0, 40.6, 33.7, 29.3, 27.6, 25.5, 22.5, 14.0, 13.7; HRMS $\mathrm{m} / z 425.1607$ (calcd for $\mathrm{C}_{21} \mathrm{H}_{28} \mathrm{CINO}_{6}$, 425.1605).

Compound 11d: The title compound was prepared according to the general $\mathrm{Yb}(\mathrm{OTf})_{3}$ catalyzed cycloaddition procedure for 15 minutes to afford a yellow oil (29 mg, 21\%); $\mathrm{R}_{f} 0.40$ (70\% hexanes /EtOAc); ${ }^{1} \mathrm{H}$ NMR $\left(600 \mathrm{MHz}, \mathrm{CDCl}_{3}\right) \delta 7.16-7.24(\mathrm{~m}, 4 \mathrm{H}), 6.99-7.03(\mathrm{~m}, 1 \mathrm{H}), 5.03$ (dd, J $=7.9,3.8 \mathrm{~Hz}, 1 \mathrm{H}), 4.19-4.25(\mathrm{~m}, 2 \mathrm{H}), 4.11-4.19(\mathrm{~m}, 2 \mathrm{H}), 3.94(\mathrm{dq}, J$ $=9.9,7.1 \mathrm{~Hz}, 1 \mathrm{H}), 3.61(\mathrm{dq}, J=9.9,7.1 \mathrm{~Hz}, 1 \mathrm{H}), 2.58(\mathrm{dt}, 13.5,4.7 \mathrm{~Hz}$, $1 \mathrm{H}$ ), 2.44 (ddd, 13.4, 12.2, $4.4 \mathrm{~Hz}, 1 \mathrm{H}$ ), 1.97 (dq, $J=13.4,4.2 \mathrm{~Hz}, 1 \mathrm{H}$ ), $1.76-1.84(\mathrm{~m}, 1 \mathrm{H}), 1.22(\mathrm{t}, J=7.0 \mathrm{~Hz}, 3 \mathrm{H}), 1.18(\mathrm{t}, J=7.0 \mathrm{~Hz}, 3 \mathrm{H}), 1.13$ $(\mathrm{t}, J=7.0 \mathrm{~Hz}, 3 \mathrm{H}) ;{ }^{13} \mathrm{C}$ NMR $\left(101 \mathrm{MHz}, \mathrm{CDCl}_{3}\right) \delta 168.4,168.1,148.3$ 127.7, 123.3, 119.5, 102.5, 74.8, 64.7, 61.9, 61.6, 30.8, 27.0, 15.1, 13.8, 13.7; HRMS $\mathrm{m} / \mathrm{z} 351.1679$ (calcd for $\mathrm{C}_{18} \mathrm{H}_{25} \mathrm{NO}_{6}, 351.1682$ ).

Compound 12a: The title compound was prepared according to the general $\mathrm{Mgl}_{2}$ catalyzed cycloaddition procedure at $0{ }^{\circ} \mathrm{C}$ for 15 minutes to afford a yellow oil (43 mg, 38\%); $\mathrm{R}_{f} 0.36$ (70\% hexanes /EtOAc); ${ }^{1} \mathrm{H}$ NMR $\left(600 \mathrm{MHz}, \mathrm{CDCl}_{3}\right) \delta 7.19-7.23(\mathrm{~m}, 2 \mathrm{H}), 6.75-6.79(\mathrm{~m}, 2 \mathrm{H}), 5.00$ (dd, J $=8.2,3.5 \mathrm{~Hz}, 1 \mathrm{H}), 4.06-4.23(\mathrm{~m}, 4 \mathrm{H}), 3.83-3.90(\mathrm{~m}, 1 \mathrm{H}), 3.77(\mathrm{~s}, 3$ H), $3.53-3.60(\mathrm{~m}, 1 \mathrm{H}), 2.57(\mathrm{dt}, J=13.5,4.7 \mathrm{~Hz}, 1 \mathrm{H}), 2.44(\mathrm{td}, J=12.6$, $4.7 \mathrm{~Hz}, 1 \mathrm{H}), 1.95(\mathrm{dq}, J=13.4,4.2 \mathrm{~Hz}, 1 \mathrm{H}), 1.73-1.83(\mathrm{~m}, 1 \mathrm{H}), 1.17$ $1.22(\mathrm{~m}, 6 \mathrm{H}), 1.13(\mathrm{t}, J=7.0 \mathrm{~Hz}, 3 \mathrm{H}) ;{ }^{13} \mathrm{C} \mathrm{NMR}\left(101 \mathrm{MHz}, \mathrm{CDCl}_{3}\right) \delta 168.3$ 168.1, 156.4, 141.3, 122.7, 112.8, 102.3, 75.2, 64.6, 61.8, 61.5, 55.4, 27.1, $37.7,15.1,13.9,13.8$; HRMS $\mathrm{m} / \mathrm{z} 381.1778$ (calcd for $\mathrm{C}_{19} \mathrm{H}_{27} \mathrm{NO}_{7}$ 381.1788).

Compound 14a: To a stirred solution of $\mathrm{Mgl}_{2}(47 \mathrm{mg}, 0.17 \mathrm{mmol})$ in $6 \mathrm{~mL}$ $\mathrm{CH}_{2} \mathrm{Cl}_{2}$ at room temperature was added $12 \mathrm{a}(130 \mathrm{mg}, 0.34 \mathrm{mmol})$. The reaction mixture was stirred for about 30 minutes at room temperature (complete consumption of starting material by TLC) then the reaction mixture was layered directly onto a silica gel column and purified by flash chromatography $(0-25 \%$ EtOAc/hexanes) to afford a yellow oil $(50 \mathrm{mg}$, $38 \%) ; \mathrm{R}_{f} 0.43\left(70 \%\right.$ hexanes /EtOAc); ${ }^{1} \mathrm{H}$ NMR (400 MHz, $\left.\mathrm{CDCl}_{3}\right) \delta 6.70$ $6.75(\mathrm{~m}, 2 \mathrm{H}), 6.58-6.64(\mathrm{~m}, 2 \mathrm{H}), 4.81(\mathrm{~s}, 1 \mathrm{H}), 4.16-4.26(\mathrm{~m}, 4 \mathrm{H}), 4.04$ (q, J=7.2 Hz, $2 \mathrm{H}), 3.73(\mathrm{~s}, 3 \mathrm{H}), 2.61-2.68(\mathrm{~m}, 2 \mathrm{H}), 2.22-2.29(\mathrm{~m}, 2$ $\mathrm{H}), 1.19$ (t, $J=7.0 \mathrm{~Hz}, 9 \mathrm{H}) ;{ }^{13} \mathrm{C}$ NMR $\left(101 \mathrm{MHz}, \mathrm{CDCl}_{3}\right) \delta 196.3,172.7$, 169.6, 153.2, 137.6, 116.9, 114.6, 68.1, 62.4, 60.5, 55.6, 28.7, 27.4, 14.1, 13.9; HRMS m/z 381.1789 (calcd for $\mathrm{C}_{19} \mathrm{H}_{27} \mathrm{NO}_{7}, 381.1788$ ).

\section{Acknowledgements}

We thank the University of Western Ontario and the Natural Sciences and Engineering Research Council of Canada for financial support. We also thank Ms. Aneta Borecki for X-ray crystallographic analysis, Dr. Mathew Willans for NMR spectrographic assistance, Mr. Doug Hairsine for high resolution mass spectroscopy, Prof. Michael A. Kerr and Dr. Andrew C. Stevens for helpful discussions (all from UWO).

[3] For reviews, see; (a) T. Seiser, T. Saget, D. N. Tran and N. Cramer Angew. Chem. Int. Ed. 2011, 50, 7740; (b) J. I. Matsuo, Tetrahedron Lett. 2014, 55, 2589; (c) F. de Nanteuil, F. De Simone, R. Frei, F. Benfatt, E. Serrano and J. Waser, Chem. Commun. 2014, 50, 10912; (d) H.-U. Reissig and R. Zimmer, Angew. Chem. Int. Ed. 2015, 54, 5009.

[4] For examples see; (a) S. Shimada, K. Saigo, H. Nakamura and M. Hasegawa, Chem. Lett. 1991, 20,1149; (b) E. A. Allart, S. D. R. Christie, G. J. Pritchard and M. R. J. Elsegood, Chem. Commun. 2009, 7339; (c) A. T. Parsons and J. S. Johnson, J. Am. Chem. Soc. 2009, 131, 14202; 
(d) M. M. A. R. Moustafa and B. L. Pagenkopf, Org. Lett. 2010, 12, 4732; (e) M. M. A. R. Moustafa, A. C. Stevens, B. P. Machin and B. L. Pagenkopf, Org. Lett. 2010, 12, 4736; (f) B. P. Machin and B. L. Pagenkopf, Synlett 2011, 2799; (g) A. C. Stevens, C. Palmer and B. L. Pagenkopf, Org. Lett. 2011, 13, 1528; (h) F. de Nanteuil and J. Waser, Angew. Chem. Int. Ed. 2013, 52, 9009; (i) R. Shenje, M. C. Martin and S. France, Angew. Chem. Int. Ed. $2014,53,13907$. (j) D. Perrotta, S. Racine, J. Vuilleumier, F. de Nanteuil, J. Waser, Org. Lett. 2015, 17, 1030.

[5] For examples, see; (a) J.-i. Matsuo, S. Sasaki, H. Tanaka and H. Ishibashi, J. Am. Chem. Soc. 2008, 130, 11600; (b) J.-i. Matsuo, S. Sasaki, T. Hoshikawa and H. Ishibashi, Org. Lett. 2009, 11, 3822; (c) J.-i. Matsuo, S. Negishi and H. Ishibashi, Tetrahedron Lett. 2009, 50, 5831; (d) J.-i. Matsuo, S. Sasaki, T. Hoshikawa and H. Ishibashi, Chem. Commun. 2010, 46, 934; (e) S. Negishi, H. Ishibashi and J.-i. Matsuo, Org. Lett. 2010, 12, 4984; (f) J.-i. Matsuo, R. Okado and H. Ishibashi, Org. Lett. 2010, 12, 3266; (g) M. Kawano, T. Kiuchi, S. Negishi, H. Tanaka, T. Hoshikawa, J.-i. Matsuo and H. Ishibashi, Angew. Chem. Int. Ed. 2013, 52, 906; (h) L. Souillart and N. Cramer, Chem. Eur. J. 2015, 21, 1863.

[6] N. Vemula, A. C. Stevens, T. B. Schon and B. L. Pagenkopf, Chem. Commun. 2014, 50, 1668

[7] See experimental section for complete list of catalyst screening

[8] See experimental section for complete optimization study.

[9] CCDC 1061592 contains the crystal data for 7c. These data can be obtained free of charge from The Cambridge Crystallographic Data Centre via www.ccdc.cam.ac.uk.

[10] No conversion to $\mathbf{5 c}$ was observed when $\mathbf{4 c}$ was exposed to $50 \mathrm{~mol} \% \mathrm{Mgl}_{2}$.

[11] CCDC 1061593 contains the crystal data for 9a. These data can be obtained free of charge from The Cambridge Crystallographic Data Centre via www.ccdc.cam.ac.uk.

[12] W. L. F. Armarego, D. D. Perrin, Purification of Laboratory Chemicals; 4th Ed.; Oxford; Boston: Butterworth Heinemann, 1996.

[13] See footnote 50 in: Y. Gao, R. M. Hanson, J. M. Klunder, S. Y. Ko, H. Masamune, K. B. Sharpless, J. Am. Chem. Soc. 1987, 109, 5765

[14] W. C. Still, M. Kahn, A. Mitra, J. Org. Chem. 1978, 43, 2923.

[15] (a) E. C. Taylor, C.P. Tseng, J. B. Rampal. J. Org. Chem. 1982, 47, 552; (b) B. Priewisch, K. Rück-Braun, J. Org. Chem. 2005, 70, 2350; (c) A. Defoin, Synthesis 2008, 760. (d) G. A. Molander and L. N. Cavalcanti, J. Org. Chem. 2012, 77, 4402. 


\section{FULL PAPER}

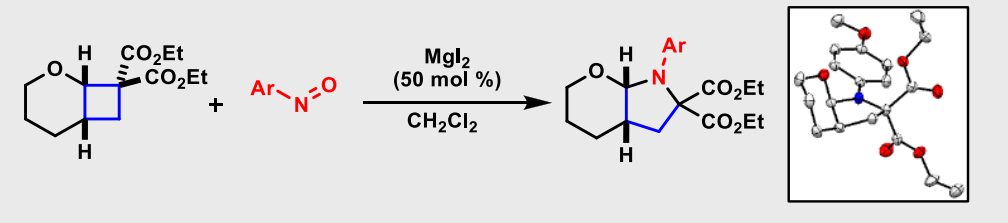

During efforts to expand the scope of Lewis acid catalyzed [4+2] cycloaddition between donor-acceptor cyclobutanes and nitrosoarenes, an unexpected formation of pyrrolidine products was discovered when $50 \mathrm{~mol} \%$ of $\mathrm{Mgl}_{2}$ was used as a Lewis acid. It was also observed that the electronics of the nitrosoarene and judicious selection of the Lewis acid catalyst have a profound effect on the regioselectivity of the reaction.

\section{Cycloadditions}

Naresh Vemula and Brian L. Pagenkopf*

Page No. - Page No.

Synthesis of Tetrahydro-1,2-oxazines and Pyrrolidines via Cycloadditions of DonorAcceptor Cyclobutanes and Nitrosoarenes

[a] Department of Chemistry 